\title{
Energy Consumption Comparison of a Novel Parallel Tracker and Its Corresponding Serial Tracker
}

\author{
Hengchun Cui, ${ }^{1,2}$ Jun Wu (D), ${ }^{1,2}$ Binbin Zhang, ${ }^{1,2}$ Liping Wang, ${ }^{1,2}$ and Tian Huang ${ }^{3,4}$ \\ ${ }^{1}$ State Key Laboratory of Tribology and Institute of Manufacturing Engineering, Department of Mechanical Engineering, \\ Tsinghua University, Beijing 100084, China \\ ${ }^{2}$ Beijing Key Lab of Precision/Ultra-Precision Manufacturing Equipment and Control, Beijing 100084, China \\ ${ }^{3}$ Key Laboratory of Mechanism Theory and Equipment Design of Ministry of Education, Tianjin University, \\ Tianjin 300354, China \\ ${ }^{4}$ School of Engineering, University of Warwick, Coventry CV4 7AL, UK
}

Correspondence should be addressed to Jun Wu; jhwu@mail.tsinghua.edu.cn

Received 30 October 2020; Revised 2 February 2021; Accepted 27 February 2021; Published 24 March 2021

Academic Editor: Adiel T. de Almeida-Filho

Copyright ( 2021 Hengchun Cui et al. This is an open access article distributed under the Creative Commons Attribution License, which permits unrestricted use, distribution, and reproduction in any medium, provided the original work is properly cited.

A novel two-axis solar tracker with parallel mechanism is proposed in this paper. A dynamic model is derived by using the virtual work principle and the consumed energy including the mechanical energy and motor energy loss is computed. Taking Beijing as the working location of the solar tracker, the energy consumptions of the parallel solar tracker and its corresponding serial solar tracker are compared based on the premise that the proposed solar tracker and its corresponding serial solar tracker have similar static stiffness. Mechanical energy consumption of the proposed tracker is reduced by $7.55 \%$ compared to the serial solar tracker. The motor energy loss of the parallel solar tracker is also significantly lower. This simple and low-energy consumption solar tracker is a good alternative to the traditional solar tracker with large energy consumption.

\section{Introduction}

As a new energy source, solar energy has certain advantages, such as energy reserves and cleanliness, which are absent in traditional fossil energy [1-3]. The solar collector is used to absorb the solar energy and the concentrator is installed on the solar tracker, which keeps the concentrator in the most efficient position for absorbing solar energy throughout the day. Solar trackers are an essential part of concentrating solar energy and photovoltaics, which can improve the amount of energy captured.

Solar trackers include passive and active trackers, and active trackers can be divided into programmed ones and sensorized ones [4]. By considering mechanical characteristics, the solar tracker can be mainly divided into single-axis solar trackers and two-axis solar trackers [5]. Single-axis trackers are mostly used on certain small solar collectors, and they are divided in vertical [6], horizontal [7], and inclined axis trackers [8]. The two-axis solar tracker can be classified into two-axis polar-mount, rotating platform dualaxis trackers and other trackers $[9,10]$. Optimal tracking of the Sun's path can be obtained by two-axis solar trackers. Thus, dual-axis trackers are more popular in most kinds of solar energy devices. However, the majority of two-axis solar trackers are designed on the basis of traditional serial mechanism [11-14]. Serial mechanism commonly requires heavy links to maintain sufficient rigidity to withstand the load of solar mirrors or panels. Moreover, reducers with a large reduction ratio are needed for the servomotor to generate substantial torque. Therefore, the tracker with serial mechanism is a heavy-duty equipment that consumes considerable energy [15].

Parallel mechanisms have high stiffness and payload to weight ratio over the serial mechanism $[16,17]$. Thus, solar trackers can also be designed based on parallel mechanism to reduce the mounting dimensions and the complexity of the system with respect to the quantity of its assembly and components [18-24]. However, the main drawback of 
parallel solar tracker is the small workspace. To overcome the drawback, some research added actuation redundancy or very long links in the parallel solar tracker [25-27]. Therefore, the system complexity is improved and the stiffness of the solar tracker with long links is reduced. In addition, the solar tracker is a low-speed mechanism and its electrical energy consumption greatly contributes to the total energy consumption. To the best of the authors' knowledge, up to now, very few studies have been conducted on the electrical energy consumption. Furthermore, only few comparison studies on the energy consumption of conventional solar tracker and parallel solar tracker are available.

In this paper, a two-axis solar tracker with parallel mechanism is proposed. The solar tracker has two kinematic chains and big workspace for tracking the sun. The mechanical and electrical energy consumptions are investigated. Moreover, the mechanical energy consumption and the energy loss caused by the resistance and inductance of the proposed solar tracker and its corresponding serial solar tracker are compared. This paper is organized as follows. The structure description and kinematics are presented in Section 2. The inverse dynamic model is established through the virtual work principle in Section 3. The calculation method of the power under different working conditions of the motor is discussed in Section 4. The calculation results and corresponding analysis are shown in Section 5, and the paper is summarized in Section 6.

\section{Structure Description and Kinematics}

2.1. Structure Description. The conventional two-axis solar tracker with serial mechanism is similar to a 2-DOF RR ( R stands for revolute joint) robot manipulator. This tracker has a vertical and a transversal pole mounted on the vertical pole. Due to the cantilever structure, the solar tracker with serial mechanism has great torque for the actuators. Based on the conventional two-axis solar tracker, this paper proposes a new two-axis solar tracker by replacing one active joint by passive joint and adding a UPS chain ( $U$ is universal joint, $P$ is prismatic joint, and $S$ is spherical joint) with one actuator to the conventional RR solar tracker, as shown in Figure 1.

Two revolute joints connect the column with the base platform and the moving platform, respectively, the universal joint connects the extendible link and the base platform, and the spherical joint connects the extendible link and the moving platform. The new solar tracker has two DOFs. The first motor drives the column around the vertical axis through a reducer, and the second motor drives the upper part of the extendible link to move along its axis. The RR chain can rotate about the axial axes of the two $R$ joints, whereas the UPS chain has no kinematic constraint to the mobile platform. Moreover, the driving torque provided by the motor can be reduced by attaching the solar mirror on the center of the moving platform.

Some solar trackers based on parallel mechanisms are also presented. The solar tracker in this paper is compared with a few solar trackers, as shown in Table $1[18,28,29]$. The solar tracker in this paper is more simple and cheaper than these solar trackers, although it is also created based on a parallel mechanism.

2.2. Motion Planning. Figure 2 shows the positions of the Sun and the Earth. Assume that the tracker is placed in Beijing. $O_{0}-X Y Z$ is the celestial reference frame with the $Z$-axis pointing to the meridian where Beijing is located. $\varphi$ is the latitude of Beijing. $\delta$ is the declination of the Sun, which can be determined by

$$
\delta=-23.45 \cos \frac{360(n+10)}{365},
$$

where $n$ is the number of days in a year starting from January $1^{\text {st. }} . T$ is the hour angle and it can be written as

$$
T=\pi\left(1-\frac{t}{12}\right),
$$

where $t=\mathrm{GMT}+08: 00$. Thus, the orientation vector of the Sun in the celestial reference frame can be expressed as

$$
\mathbf{s}=\left[\begin{array}{ccc}
\cos T & -\sin T & 0 \\
\sin T & \cos T & 0 \\
0 & 0 & 1
\end{array}\right]\left[\begin{array}{ccc}
\sin \delta & 0 & \cos \delta \\
0 & 1 & 0 \\
-\cos \delta & 0 & \sin \delta
\end{array}\right] \mathbf{e}_{3}=\left[\begin{array}{c}
\cos \delta \cos T \\
\cos \delta \sin T \\
\sin \delta
\end{array}\right]
$$

where $\mathbf{e}_{3}=\left[\begin{array}{lll}0 & 0 & 1\end{array}\right]^{T} . O-x y z$ is the horizontal reference frame whose $x$-axis points south, and $O$ represents the center of revolute joint connected to the base of the tracker as shown in Figure 3. Solar direction can also be described by altitude and azimuth. The generalized coordinate $\alpha$ is the supplementary angle of the azimuth of the Sun. To facilitate the description of the posture of the moving platform, the generalized coordinate $\beta$ is the complementary angle of the solar altitude. The generalized coordinate is defined as $\mathbf{q}=\left[\begin{array}{ll}\alpha & \beta\end{array}\right]^{T}$.

Figure 3 shows the schematic diagram of the new twoaxis solar tracker, where $O^{\prime}$ represents the center of revolute joint connected to the moving platform, and $B$ and $O^{\prime \prime}$ are the centers of the spherical joint and universal joint, respectively. A body-fixed coordinate system $O^{\prime}-x^{\prime} y^{\prime} z^{\prime}$ is established, where $y^{\prime}$-axis is the rotation axis of the revolute joint and $z^{\prime}$-axis is parallel to the normal direction of the moving platform. A body-fixed coordinate system $O^{\prime \prime}-x^{\prime \prime} y^{\prime \prime} z^{\prime \prime}$ is then established as well, where $z^{\prime \prime}$-axis is coincident with UPS limb, and $y^{\prime \prime}$-axis is parallel to the horizontal rotation axis of the universal joint. The orientation of $O^{\prime}-x^{\prime} y^{\prime} z^{\prime}$ with respect to $O-x y z$ can be described as 


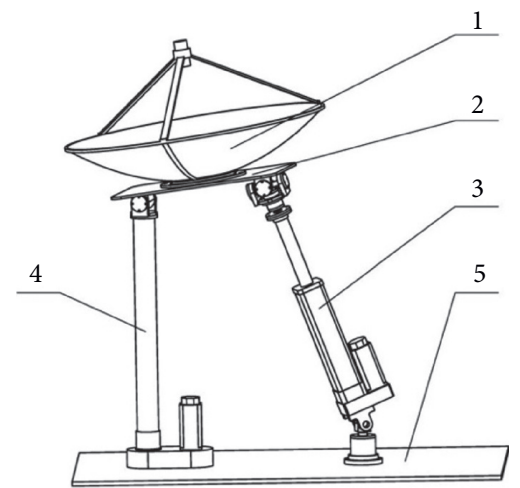

FIgURE 1: 3D model. 1-solar mirror, 2-moving platform, 3-extendible link, 4-column, 5-base platform.

TABLE 1: Comparison with other topologies.

\begin{tabular}{|c|c|c|c|c|}
\hline Торс & UPS-RR & U-PRU-PUS & U-2PUS & U-3PSS \\
\hline Cha & $\begin{array}{l}\text { High stiffness, relatively } \\
\text { simple structure, low } \\
\text { equipment cost, enough } \\
\text { workspace }\end{array}$ & $\begin{array}{c}\text { High stiffness, insufficient } \\
\text { workspace, relatively complex } \\
\text { structure, relatively high } \\
\text { equipment cost }\end{array}$ & $\begin{array}{c}\text { High stiffness, large } \\
\text { workspace, relatively } \\
\text { complex structure, relatively } \\
\text { high equipment cost }\end{array}$ & $\begin{array}{c}\text { High stiffness, large } \\
\text { workspace, relatively } \\
\text { complex structure, relatively } \\
\text { high equipment cost }\end{array}$ \\
\hline
\end{tabular}

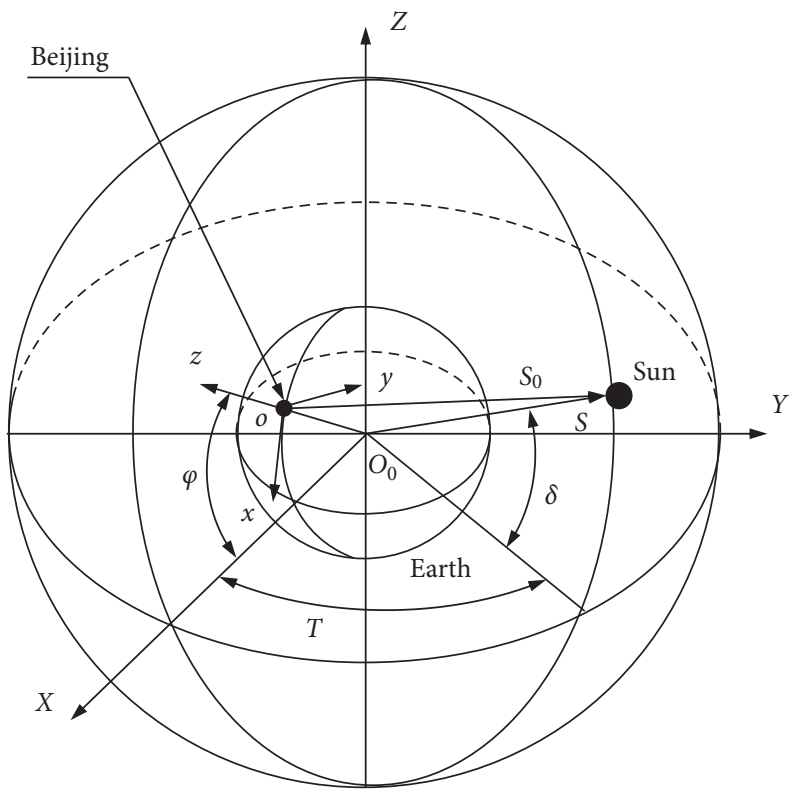

Figure 2: Celestial reference frame.

$$
\begin{aligned}
\mathbf{R}_{p} & =\operatorname{Rot}(z, \alpha) \operatorname{Rot}\left(y^{\prime}, \beta\right) \\
& =\left[\begin{array}{ccc}
\cos \alpha \cos \beta & -\sin \alpha & \cos \alpha \sin \beta \\
\sin \alpha \cos \beta & \cos \alpha & \sin \alpha \sin \beta \\
-\sin \beta & 0 & \cos \beta
\end{array}\right] .
\end{aligned}
$$

Thus, the orientation vector of the Sun in the horizontal reference frame can be expressed as

$$
\mathbf{s}_{O}=\mathbf{R}_{p} \mathbf{e}_{3}=\left[\begin{array}{c}
\cos \alpha \sin \beta \\
\sin \alpha \sin \beta \\
\cos \beta
\end{array}\right] .
$$

The distance between the Sun and the Earth is much larger than the radius of the Earth. Thus, the distances of the Sun from the origins of two reference frames are 


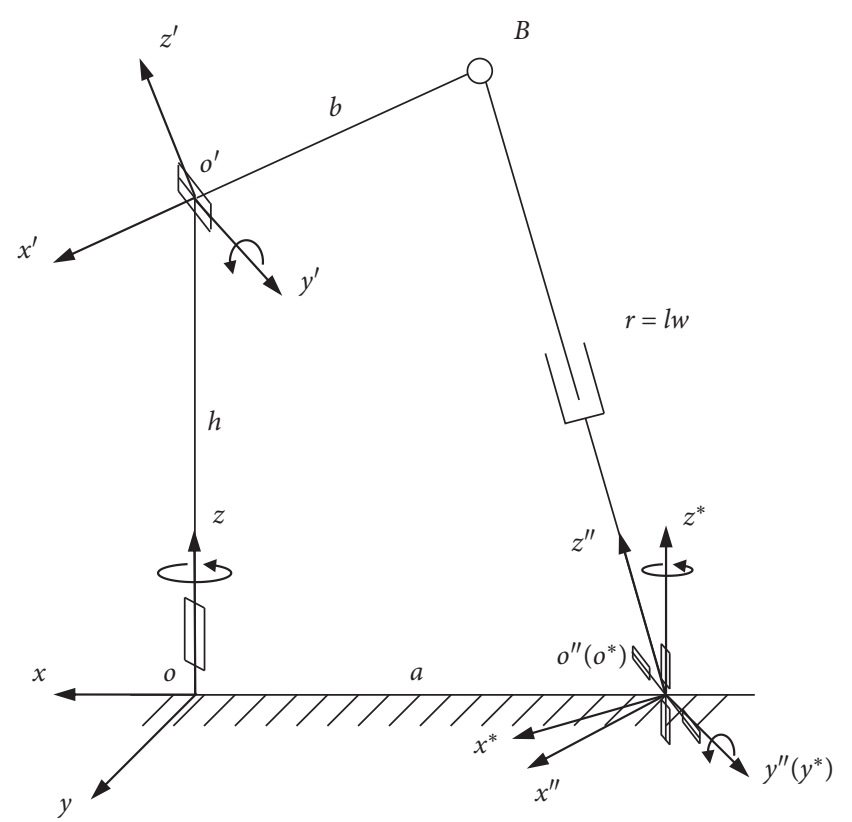

FIGURE 3: Schematic diagram of parallel solar tracker.

approximately equal, and the two orientation vectors should be equal when they are expressed in the same reference frame. Thus, the relationship between $\mathbf{s}$ and $\mathbf{s}_{O}$ can be written as

$$
\mathbf{s}=\boldsymbol{\operatorname { R o t }}\left(Y, \frac{\pi}{2}-\varphi\right) \mathbf{s}_{O}
$$

$\alpha$ and $\beta$ can be expressed as

$$
\begin{aligned}
& \alpha=\arctan \frac{\cos \delta \sin T}{\sin \varphi \cos \delta \cos T-\cos \varphi \sin \delta}, \\
& \beta=\arccos (\sin \varphi \sin \delta+\cos \varphi \cos \delta \cos T) .
\end{aligned}
$$

Weather will affect the refractive index of the Earth's atmosphere. Thus, it is more accurate to optimize the trajectory of the solar tracker by taking the weather conditions into account $[30,31]$. In this paper, it is assumed that the solar tracker is used in Beijing, where the climate is relatively dry throughout the year. Therefore, the impact of climate is not considered in this paper.

2.3. Inverse Kinematics. In $O-x y z$, the closed-loop constraint equation can be expressed as

$$
\begin{aligned}
& \mathbf{r}=l \mathbf{w}, \\
& \mathbf{r}=\mathbf{h}+\mathbf{b}-\mathbf{a},
\end{aligned}
$$

where $\mathbf{r}$ is the position vector of point $B$ in $O^{\prime \prime}-x^{\prime \prime} y^{\prime \prime} z^{\prime \prime} ; l$ and $\mathbf{w}$ are the length and unit vector of the extendible link; $\mathbf{h}$ and $\mathbf{a}$ are the position vectors of $O^{\prime}$ and $O^{\prime \prime}$ in $O-x y z ; \mathbf{b}=\mathbf{R}_{p} \mathbf{b}^{\prime}$; and $\mathbf{b}^{\prime}$ is the position vector of point $B$ in $O^{\prime}-x^{\prime} y^{\prime} z^{\prime}$. The length of the extendible link can be expressed as

$$
l=\|\mathbf{r}\|=\sqrt{\left(\mathbf{h}+\mathbf{R}_{p} \mathbf{b}^{\prime}-\mathbf{a}\right)^{T}\left(\mathbf{h}+\mathbf{R}_{p} \mathbf{b}^{\prime}-\mathbf{a}\right)} .
$$

The unit vector of the extendible link can be obtained as

$$
\mathbf{w}=\frac{\mathbf{r}}{l}=\frac{\mathbf{h}+\mathbf{R}_{p} \mathbf{b}^{\prime}-\mathbf{a}}{l} .
$$

The rotational matrix of the extendible link can be expressed as $\mathbf{R}=\boldsymbol{\operatorname { R o t }}\left(z, \theta_{1}\right) \boldsymbol{\operatorname { R o t }}\left(y^{*}, \theta_{2}\right)$, where $\theta_{1}=\arctan$ $\left(\mathbf{w}^{T} \mathbf{e}_{2} / \mathbf{w}^{T} \mathbf{e}_{1}\right)$ and $\theta_{2}=\arccos \left(\mathbf{w}^{T} \mathbf{e}_{3} / \sqrt{\mathbf{w}^{T} \mathbf{w}}\right)$, which will be used to calculate the generalized force in Section 4 . The rotation angle of the column is $\alpha$. Thus, the solution of the inverse kinematics has been found.

The position of the mass center of each component can be written as

$$
\begin{aligned}
& \mathbf{r}_{c 1}=0.5 \mathbf{h}, \\
& \mathbf{r}_{c p}=\mathbf{h}+\mathbf{R}_{p} \mathbf{r}_{p}^{\prime}, \\
& \mathbf{r}_{c 2}=\mathbf{a}+\left(l-0.5 l_{2}\right) \mathbf{w}, \\
& \mathbf{r}_{c 3}=\mathbf{a}+0.5 l_{3} \mathbf{w},
\end{aligned}
$$

where $\mathbf{r}_{p}^{\prime}$ is the centroid position of the moving platform, including the parabolic dish concentrators, in $O^{\prime}-x^{\prime} y^{\prime} z^{\prime}$, and $l_{2}$ and $l_{3}$ are the lengths of the upper and the lower parts of the extendible link, respectively.

2.4. Velocity Analysis. Link $O O^{\prime}$ only rotates around the vertical axis, and its angular velocity is equal to $\dot{\alpha} \mathbf{e}_{3}$. The Jacobian submatrix that represents the mapping from output angular velocity to the angular velocity of link $O O^{\prime}$ is $\mathbf{J}_{\boldsymbol{\omega} 1}=\left[\begin{array}{ll}\mathbf{e}_{3} & 0\end{array}\right]$, and the Jacobian submatrix associated with the linear velocity is equal to zero.

Substituting $\mathbf{b}=\mathbf{R}_{p} \mathbf{b}^{\prime}$ into equation (8b) and then differentiating it with respect to time lead to

$$
\dot{\mathbf{r}}=\frac{\mathrm{d} \mathbf{R}_{p} \mathbf{b}^{\prime}}{\mathrm{d} t}=\frac{\partial \mathbf{R}_{p_{p}}}{\partial \alpha} \mathbf{b}^{\prime} \dot{\alpha}+\frac{\partial \mathbf{R}_{p}}{\partial \beta} \mathbf{b}^{\prime} \dot{\beta}=\left[\frac{\partial \mathbf{R}_{p}}{\partial \alpha} \mathbf{b}^{\prime} \frac{\partial \mathbf{R}_{p}}{\partial \beta} \mathbf{b}^{\prime}\right]\left[\begin{array}{c}
\dot{\alpha} \\
\dot{\beta}
\end{array}\right]=\mathbf{J}_{r} \dot{\mathbf{q}},
$$

where $\mathbf{J}_{r}$ is the Jacobian matrix associated with the velocity of point $B$. By taking the time derivative of equations (8a) and (8b), the following equation can be obtained:

$$
\dot{\mathbf{r}}=i_{\mathbf{w}}+l \omega \times \mathbf{w} .
$$

Taking the dot product with $\mathbf{w}$ on both sides of equation (13) yields

$$
\begin{aligned}
\mathbf{w}^{T} \dot{\mathbf{r}} & =i_{\mathbf{w}^{T}} \mathbf{w}+l \mathbf{w}^{T}(\boldsymbol{w} \times \mathbf{w})=\dot{l} \\
& =\mathbf{w}^{T} \mathbf{J}_{r} \dot{\mathbf{q}} .
\end{aligned}
$$

Substituting equation (14) into equation (13) yields

$$
\mathbf{w} \times \boldsymbol{w}=\frac{i \mathbf{w}-\dot{\mathbf{r}}}{l}=\frac{\left(\mathbf{w} \mathbf{w}^{\mathrm{T}}-\mathbf{E}_{3}\right) \mathbf{J}_{\mathbf{r}} \dot{\mathbf{q}}}{l},
$$


where $\mathbf{w}=\left[\begin{array}{lll}w_{x} & w_{y} & w_{z}\end{array}\right]^{T}$ and $[\mathbf{w} \times]=\left[\begin{array}{ccc}0 & -w_{z} & w_{y} \\ w_{z} & 0 & -w_{x} \\ -w_{y} & w_{x} & 0\end{array}\right]$

$\boldsymbol{\omega}$ cannot be solved directly by equation (15). The extendible link can rotate around the $y^{*}$-axis and the $z^{*}$-axis at the same time. Therefore, $\boldsymbol{\omega}$ must be in the plane $y^{*} O^{*} z^{*}$ according to the vector synthesis rule. The transformation process of coordinate system shows that $\mathbf{w}$ is in plane $x^{*} O^{*} z^{*}$. Thus, the projection of $\mathbf{w}$ on the $x^{*}$-axis is the horizontal projection of $\mathbf{w}$. Therefore, the following equation can be obtained:

$$
\left(\mathbf{D}[\mathbf{w} \times]+\mathbf{e}_{3} \mathbf{w}^{T} \mathbf{D}^{T}\right) \mathbf{w}=\frac{1}{l} \mathbf{D}\left(\mathbf{w} \mathbf{w}^{T}-\mathbf{E}_{3}\right) \mathbf{J}_{r} \dot{\mathbf{q}},
$$

where $\mathbf{D}=\mathbf{E}_{3}-\mathbf{e}_{3} \mathbf{e}_{3}^{T}$. Thus, the angular velocity of the extendible link can be written as

$$
\boldsymbol{\omega}=\frac{1}{l}\left(\mathbf{D}[\mathbf{w} \times]+\mathbf{e}_{3} \mathbf{w}^{T} \mathbf{D}^{T}\right)^{-1} \mathbf{D}\left(\mathbf{w} \mathbf{w}^{T}-\mathbf{E}_{3}\right) \mathbf{J}_{r} \dot{\mathbf{q}}=\mathbf{J}_{\boldsymbol{\omega}} \dot{\mathbf{q}}
$$

where $\mathbf{J}_{\boldsymbol{\omega}}$ represents the mapping from output angular velocity to the angular velocity of the extendible link. Taking the time derivative of equation (11c) leads to

$$
\begin{aligned}
\dot{\mathbf{r}}_{C 2} & =i_{\mathbf{w}}+\left(l-0.5 l_{2}\right) \mathbf{w} \times \mathbf{w} \\
& =\left(\mathbf{w} \mathbf{w}^{T} \mathbf{J}_{r}-\left(l-0.5 l_{2}\right)[\mathbf{w} \times] \mathbf{J}_{\boldsymbol{\omega}}\right) \dot{\mathbf{q}}=\mathbf{J}_{C 2} \dot{\mathbf{q}},
\end{aligned}
$$

where $\mathbf{J}_{\mathrm{C} 2}$ represents the mapping from output angular velocity to the velocity of upper part's mass center. By taking the time derivative of equation (11d), the mass center velocity of the lower part of the extendible link can be written as

$$
\dot{\mathbf{r}}_{C 3}=0.5 l_{3} \mathbf{w} \times \mathbf{w}=-0.5 l_{3}[\mathbf{w} \times] \mathbf{J}_{\omega} \dot{\mathbf{q}}=\mathbf{J}_{C 3} \dot{\mathbf{q}},
$$

where $\mathbf{J}_{C 3}$ represents the mapping from output angular velocity to the velocity of lower part's mass center. The rotational matrix of the moving platform is expressed by equation (4). Since $\left[\boldsymbol{\omega}_{p} \times\right]=\dot{\mathbf{R}}_{p} \mathbf{R}_{p}^{T}$, the angular velocity of the moving platform can be expressed as

$$
\boldsymbol{\omega}_{p}=\left[\begin{array}{c}
\mathbf{e}_{3}^{T}\left[\boldsymbol{\omega}_{p} \times\right] \mathbf{e}_{2} \\
\mathbf{e}_{1}^{T}\left[\boldsymbol{\omega}_{p} \times\right] \mathbf{e}_{3} \\
\mathbf{e}_{2}^{T}\left[\boldsymbol{\omega}_{p} \times\right] \mathbf{e}_{1}
\end{array}\right]=\left[\begin{array}{cc}
\mathbf{e}_{3}^{T} \frac{\partial \mathbf{R}_{p}}{\partial \alpha} \mathbf{R}_{p}^{T} \mathbf{e}_{2} & \mathbf{e}_{3}^{T} \frac{\partial \mathbf{R}_{p}}{\partial \beta} \mathbf{R}_{p}^{T} \mathbf{e}_{2} \\
\mathbf{e}_{1}^{T} \frac{\partial \mathbf{R}_{p}}{\partial \alpha} \mathbf{R}_{p}^{T} \mathbf{e}_{3} & \mathbf{e}_{1}^{T} \frac{\partial \mathbf{R}_{p}}{\partial \beta} \mathbf{R}_{p}^{T} \mathbf{e}_{3} \\
\mathbf{e}_{2}^{T} \frac{\partial \mathbf{R}_{p}}{\partial \alpha} \mathbf{R}_{p}^{T} \mathbf{e}_{1} & \mathbf{e}_{2}^{T} \frac{\partial \mathbf{R}_{p}}{\partial \beta} \mathbf{R}_{p}^{T} \mathbf{e}_{1}
\end{array}\right] \dot{\mathbf{q}}=\mathbf{J}_{\boldsymbol{\omega} p} \dot{\mathbf{q}},
$$

where $\mathbf{J}_{\boldsymbol{\omega} p}$ represents the mapping from output angular velocity to the angular velocity of the moving platform. Differentiating equation (11b) with respect to time yields

$$
\dot{\mathbf{r}}_{C p}=\dot{\mathrm{R}}_{p} \mathbf{r}_{p}^{\prime}=\left[\frac{\partial \mathbf{R}_{p}}{\partial \alpha} \mathbf{r}_{p}^{\prime} \frac{\partial \mathbf{R}_{p}}{\partial \beta} \mathbf{r}_{p}^{\prime}\right]\left[\begin{array}{c}
\dot{\alpha} \\
\dot{\beta}
\end{array}\right]=\mathbf{J}_{C p} \dot{\mathbf{q}},
$$

where $\mathbf{J}_{C p}$ represents the mapping from output angular velocity to the velocity of the moving platform.

In this paper, the lead of the screw pair of the extendible link is $1 \mathrm{~cm}$. The Jacobian matrix that represents the mapping from output angular velocity to the rotation velocity of the rotors without reducers can be written as

$$
\mathbf{J}=\left[\begin{array}{cc}
1 & 0 \\
0 & 200 \pi
\end{array}\right]\left[\begin{array}{cc}
1 & 0 \\
\frac{\partial l}{\partial \alpha} & \frac{\partial l}{\partial \beta}
\end{array}\right]=\left[\begin{array}{cc}
1 & 0 \\
0 & 200 \pi
\end{array}\right]\left[\begin{array}{c}
{ }^{2} \mathbf{e}_{1}^{T} \\
\mathbf{w}^{T} \mathbf{J}_{r}
\end{array}\right] .
$$

\section{Inverse Dynamic Model}

3.1. Acceleration Analysis. The centroid linear acceleration of link $O O^{\prime}$ is equal to zero and the angular acceleration can be written as $\dot{\boldsymbol{\omega}}_{1}=\ddot{\alpha} \mathbf{e}_{3}$. Taking the time derivative of equation (18) leads to

$$
\dot{\boldsymbol{\omega}}=\dot{\mathbf{J}}_{\boldsymbol{\omega}} \dot{\mathbf{q}}+\mathbf{J}_{\boldsymbol{\omega}} \ddot{\mathbf{q}}=\frac{1}{l}\left(\mathbf{D}[\mathbf{w} \times]+\mathbf{e}_{3} \mathbf{w}^{T} \mathbf{D}^{T}\right)^{-1}\left(\begin{array}{c}
\mathbf{D}(\boldsymbol{\omega} \times \mathbf{w}) \mathbf{w}^{T} \mathbf{J}_{r}+\mathbf{D} \mathbf{w}(\boldsymbol{\omega} \times \mathbf{w})^{T} \mathbf{J}_{r}+\mathbf{D}\left(\mathbf{w} \mathbf{w}^{T}-\mathbf{E}_{3}\right) \dot{J}_{r} \\
-\left(\mathbf{D}[(\boldsymbol{\omega} \times \mathbf{w}) \times]+\mathbf{e}_{3}(\boldsymbol{\omega} \times \mathbf{w})^{T} \mathbf{D}^{T}\right) \mathbf{J}_{\boldsymbol{\omega}}
\end{array}\right) \dot{\mathbf{q}}+\mathbf{J}_{\boldsymbol{\omega}} \ddot{\mathbf{q}}
$$

where $\dot{j}_{r}=\left[\left(\partial^{2} \mathbf{R}_{p} / \partial \alpha^{2}\right) \mathbf{b}^{\prime}\left(\partial^{2} \mathbf{R}_{p} / \partial \alpha \partial \beta\right) \mathbf{b}^{\prime}\left(\partial^{2} \mathbf{R}_{p} / \partial \alpha \partial \beta\right)\right.$

$\left.\mathbf{b}^{\prime}\left(\partial^{2} \mathbf{R}_{p} / \partial \beta^{2}\right) \mathbf{b}^{\prime}\right]\left[\begin{array}{cc}\dot{\mathbf{q}} & 0 \\ 0 & \dot{\mathbf{q}}\end{array}\right]$.
By taking the time derivative of equation (19), the mass center acceleration of the upper part of extendible link can be written as

$$
\begin{aligned}
\ddot{\mathbf{r}}_{C 2} & =\dot{\mathbf{J}}_{C 2} \dot{\mathbf{q}}+\mathbf{J}_{C 2} \ddot{\mathbf{q}} \\
& =\left(\begin{array}{c}
(\boldsymbol{\omega} \times \mathbf{w}) \mathbf{w}^{T} \mathbf{J}_{r}+\mathbf{w}(\boldsymbol{\omega} \times \mathbf{w})^{T} \mathbf{J}_{r}+\mathbf{w w}^{T} \dot{\mathbf{J}}_{r}-\mathbf{w}^{T} \mathbf{J}_{r} \dot{\mathbf{q}}[\mathbf{w} \times] \mathbf{J}_{\boldsymbol{\omega}} \\
-\left(l-0.5 l_{2}\right)[(\boldsymbol{\omega} \times \mathbf{w}) \times] \mathbf{J}_{\boldsymbol{\omega}}-\left(l-0.5 l_{2}\right)[\mathbf{w} \times] \dot{\mathbf{J}}_{\boldsymbol{\omega}}
\end{array}\right) \dot{\mathbf{q}}+\mathbf{J}_{C 2} \ddot{\mathbf{q}} .
\end{aligned}
$$


Based on equation (20), the mass center acceleration of the lower part can be written as

$$
\begin{aligned}
\ddot{\mathbf{r}}_{C 3}=\dot{\mathbf{J}}_{C 3} \dot{\mathbf{q}}+\mathbf{J}_{C 3} \ddot{\mathbf{q}}= & -0.5 l_{3}[(\boldsymbol{\omega} \times \mathbf{w}) \times] \mathbf{J}_{\boldsymbol{\omega}} \dot{\mathbf{q}}-0.5 l_{3}[\mathbf{w} \times] \dot{\mathbf{J}}_{\boldsymbol{\omega}} \dot{\mathbf{q}} \\
& +\mathbf{J}_{C 3} \ddot{\mathbf{q}} .
\end{aligned}
$$

Differentiating equation (21) with respect to time leads

$$
\dot{\boldsymbol{\omega}}_{p}=\dot{\mathbf{J}}_{\boldsymbol{\omega} p} \dot{\mathbf{q}}+\mathbf{J}_{\boldsymbol{\omega} p} \ddot{\mathbf{q}}=\left[\begin{array}{cccc}
\mathbf{e}_{3}^{T} \frac{\partial^{2} \mathbf{R}_{p}}{\partial \alpha^{2}} \mathbf{R}_{p}^{T} \mathbf{e}_{2} & \mathbf{e}_{3}^{T} \frac{\partial^{2} \mathbf{R}_{p}}{\partial \alpha} \mathbf{R}_{p}^{T} \mathbf{e}_{2} & \mathbf{e}_{3}^{T} \frac{\partial^{2} \mathbf{R}_{p}}{\partial \alpha} \mathbf{R}_{p}^{T} \mathbf{e}_{2} & \mathbf{e}_{3}^{T} \frac{\partial^{2} \mathbf{R}_{p}}{\partial \beta^{2}} \mathbf{R}_{p}^{T} \mathbf{e}_{2} \\
\mathbf{e}_{1}^{T} \frac{\partial^{2} \mathbf{R}_{p}}{\partial \alpha^{2}} \mathbf{R}_{p}^{T} \mathbf{e}_{3} & \mathbf{e}_{1}^{T} \frac{\partial^{2} \mathbf{R}_{p}}{\partial \alpha \partial \beta} \mathbf{R}_{p}^{T} \mathbf{e}_{3} & \mathbf{e}_{1}^{T} \frac{\partial^{2} \mathbf{R}_{p}}{\partial \alpha} \mathbf{R}_{p}^{T} \mathbf{e}_{3} & \mathbf{e}_{1}^{T} \frac{\partial^{2} \mathbf{R}_{p}}{\partial \beta^{2}} \mathbf{R}_{p}^{T} \mathbf{e}_{3} \\
\mathbf{e}_{2}^{T} \frac{\partial^{2} \mathbf{R}_{p}}{\partial \alpha^{2}} \mathbf{R}_{p}^{T} \mathbf{e}_{1} & \mathbf{e}_{2}^{T} \frac{\partial^{2} \mathbf{R}_{p}}{\partial \alpha} \mathbf{R}_{p}^{T} \mathbf{e}_{1} & \mathbf{e}_{2}^{T} \frac{\partial^{2} \mathbf{R}_{p}}{\partial \alpha} \mathbf{R}_{p}^{T} \mathbf{e}_{1} & \mathbf{e}_{2}^{T} \frac{\partial^{2} \mathbf{R}_{p}}{\partial \beta^{2}} \mathbf{R}_{p}^{T} \mathbf{e}_{1}
\end{array}\right]\left[\begin{array}{cc}
\dot{\mathbf{q}} & 0 \\
0 & \dot{\mathbf{q}}
\end{array}\right] \dot{\mathbf{q}}+\mathbf{J}_{\boldsymbol{\omega} p} \ddot{\mathbf{q}}
$$

Taking the time derivative of equation (22) yields

$\ddot{\mathbf{r}}_{C p}=\dot{\mathbf{J}}_{C p} \dot{\mathbf{q}}+\mathbf{J}_{C p} \ddot{\mathbf{q}}=\left[\frac{\partial^{2} \mathbf{R}_{p}}{\partial \alpha^{2}} \mathbf{r}_{p}^{\prime} \frac{\partial^{2} \mathbf{R}_{p}}{\partial \alpha \partial \beta} \mathbf{r}_{p}^{\prime} \frac{\partial^{2} \mathbf{R}_{p}}{\partial \alpha \partial \beta} \mathbf{r}_{p}^{\prime} \frac{\partial^{2} \mathbf{R}_{p}}{\partial \beta^{2}} \mathbf{r}_{p}^{\prime}\right]\left[\begin{array}{cc}\dot{\mathbf{q}} & 0 \\ 0 & \dot{\mathbf{q}}\end{array}\right] \dot{\mathbf{q}}+\mathbf{J}_{C p} \ddot{\mathbf{q}}$

3.2. Dynamic Model. The generalized force imposing on link $O O^{\prime}$ can be written as

$$
\mathbf{Q}_{1}=\left[\begin{array}{c}
m_{1} \mathbf{g} \\
\mathbf{T}_{1}-\ddot{\alpha} \mathbf{R}_{1} \mathbf{I}_{1} \mathbf{R}_{1}^{T} \mathbf{e}_{3}-\dot{\alpha}^{2} \mathbf{e}_{3} \times\left(\mathbf{R}_{1} \mathbf{I}_{1} \mathbf{R}_{1}^{T} \mathbf{e}_{3}\right)
\end{array}\right],
$$

where $\mathbf{R}_{1}=\boldsymbol{R o t}(z, \alpha)$ and $\mathbf{T}_{1}$ is the friction moment. The forces and moments of the upper part and lower part of the extendible link can be written as

$$
\mathbf{Q}_{i}=\left[\begin{array}{c}
m_{i}\left(\mathbf{g}-\ddot{r}_{C i}\right) \\
\mathbf{T}_{i}-R I_{i} \mathbf{R}^{\mathbf{T}} \dot{\boldsymbol{\omega}}-\boldsymbol{\omega} \times\left(\mathbf{R I}_{i} \mathbf{R}^{\mathbf{T}} \boldsymbol{\omega}\right)
\end{array}\right], \quad i=2,3,
$$

where $\mathbf{T}_{i}$ is the friction moment. The force and moment imposing on the moving platform can be written as

$$
\mathbf{Q}_{p}=\left[\begin{array}{c}
m_{p}\left(\mathbf{g}-\ddot{r}_{C p}\right) \\
\mathbf{T}_{p}-\mathbf{R}_{p} \mathbf{I}_{p} \mathbf{R}_{p}^{T} \dot{\boldsymbol{\omega}}-\boldsymbol{\omega}_{p} \times\left(\mathbf{R}_{p} \mathbf{I}_{p} \mathbf{R}_{p}^{T} \boldsymbol{\omega}_{p}\right)
\end{array}\right],
$$

where $\mathbf{T}_{p}$ is the friction moment. The friction moment in this paper adopts Stribeck model. It can be expressed as

$$
\mathbf{T}_{i}=\left[\mathbf{T}_{e i}+\left(\mathbf{T}_{s i}-\mathbf{T}_{e i}\right) e^{-\left|v_{i} / v_{s i}\right|^{\delta}}\right] \cdot \operatorname{sgn}\left(v_{i}\right)+\beta_{v i} v_{i}, \quad i=1,2,3, p,
$$

where $\mathbf{T}_{e i}$ is the amplitude of Coulomb friction moment, $\mathbf{T}_{s i}$ is the static friction moment, $v_{i}$ is the relative velocity, $v_{s i}$ is the velocity amplitude, and $\beta_{v i}$ is viscous friction coefficient. The principle of virtual work states that

$$
\left[\delta \alpha \frac{\delta l}{200 \pi}\right] \boldsymbol{\tau}^{p}+\delta \mathbf{q}_{p}^{T} \mathbf{Q}_{p}+\sum_{i=1}^{3} \delta \mathbf{q}_{i}^{T} \mathbf{Q}_{i}=\delta \mathbf{q}^{T}\left(\mathbf{J}^{T} \boldsymbol{\tau}^{p}+\mathbf{J}_{p}^{T} \mathbf{Q}_{p}+\sum_{i=1}^{3} \mathbf{J}_{i}^{T} \mathbf{Q}_{i}\right)=0
$$


where $\tau^{p}$ represents the driving torque of the parallel solar tracker. Thus, the driving forces provided by the servomotors can be written as

$$
\boldsymbol{\tau}^{p}=-\mathbf{J}^{-T}\left(\left[\begin{array}{c}
\mathbf{J}_{C p} \\
\mathbf{J}_{\boldsymbol{\omega} p}
\end{array}\right]^{T} \mathbf{Q}_{p}+\left[\begin{array}{c}
\mathbf{J}_{C 1} \\
0
\end{array}\right]^{T} \mathbf{Q}_{1}+\left[\begin{array}{c}
\mathbf{J}_{C 2} \\
\mathbf{J}_{\boldsymbol{\omega}}
\end{array}\right]^{T} \mathbf{Q}_{2}+\left[\begin{array}{c}
\mathbf{J}_{C 3} \\
\mathbf{J}_{\boldsymbol{\omega}}
\end{array}\right]^{T} \mathbf{Q}_{3}\right)
$$

A corresponding serial solar tracker is constructed when the extendible link of the parallel solar tracker is removed, and the passive joint of the constant length link connected to the moving platform is replaced by an active joint. The parallel solar tracker and its corresponding serial solar tracker have the same motion to track the Sun. Thus, the driving force of the corresponding serial solar tracker can be written as

$$
\boldsymbol{\tau}^{s}=-\left[\begin{array}{c}
\mathbf{J}_{C p} \\
\mathbf{J}_{\omega p}
\end{array}\right]^{T} \mathbf{Q}_{p}-\left[\begin{array}{c}
\mathbf{J}_{C 1} \\
\mathbf{0}
\end{array}\right]^{T} \mathbf{Q}_{1},
$$

where $\tau^{s}$ represents the driving torque of the serial solar tracker.

\section{Energy Consumption}

The total energy consumption includes the mechanical energy and the electrical energy caused by the resistance and inductance of the motor. The electrical energy consumption greatly contributes to the total energy consumption for the low-speed and big-torque solar tracker. Thus, electrical energy is considered to compute the total energy consumption. The total power consumed by the motor mainly includes resistive power loss $P_{R}$, inductive power loss $P_{L}$, and the power used to produce electromotive force $P_{E}$ [32]. Namely,

$$
P=P_{R}+P_{L}+P_{E}=I^{2} R+I \dot{I} L+V_{E} I,
$$

where $R$ is the motor winding resistance and $L$ is the motor inductance coefficient. The motor operates stably at low speed, and the current is proportional to torque. The output torque of servomotor is small. A gearbox is generally installed, and the current can be written as

$$
I=\frac{\tau}{K_{T} r}
$$

where $K_{T}$ represents the torque sensitivity factor and $r$ represents the reduction ratio. The motor electromotive potential $V_{E}$ can be expressed as

$$
V_{E}=K_{E} r \dot{\theta},
$$

where $K_{E}$ is the back electromotive force constant and $\dot{\theta}$ is the angular velocity of the motor, which can be obtained by using the Jacobian matrix and reduction ratio. The power used to produce electromotive force can be expressed as

$$
P_{E}=\frac{K_{E}}{K_{T}} \tau \dot{\theta}=\frac{K_{E}}{K_{T}} P_{M} \approx P_{M}
$$

where $P_{M}$ represents the mechanical power. Generally, $P_{E}$ is slightly larger than $P_{M}$ because the former contains not only $P_{M}$ but also a part of the iron loss.

Due to the low speed and acceleration of the solar tracker, the driving torque of the motor is very close to the load and the motor sometimes operates as a generator with $P_{M}$ being negative. Thus, the solar tracker maybe input mechanical energy to its motors and the motors do not provide mechanical energy to the solar tracker. For a threephase motor, the total power is

$$
P= \begin{cases}0, & \text { if } \tau \dot{\theta}<0, \\ \frac{3 \tau}{K_{T}^{2} r^{2}}(\tau R+\dot{\tau} L)+\frac{3 K_{E}}{K_{T}} \tau \dot{\theta}, & \text { if } \tau \dot{\theta} \geq 0 .\end{cases}
$$

The mechanical power of the tracker can be expressed as

$$
P_{M t}=\sum_{i=1,2} \max \left\{0, \tau_{i} \dot{\theta}_{i}\right\} \text {. }
$$

The total power of the tracker can be written as

$$
P_{t}= \begin{cases}0, & \text { if } \tau \dot{\theta}<0, \\ \sum_{i=1,2}\left[\frac{3 \tau_{i}}{K_{T i}^{2} r_{i}^{2}}\left(\tau_{i} R_{i}+\dot{\tau}_{i} L_{i}\right)+\frac{3 K_{E i}}{K_{T i}} \dot{\tau}_{i} \dot{\theta}_{i}\right], & \text { if } \tau \dot{\theta} \geq 0 .\end{cases}
$$

The motor winding resistance, motor inductance coefficient, torque sensitivity factor, and back electromotive force constant are related to the motor property.

When both motors work normally, the mechanical power of the parallel solar tracker can be expressed as

$$
P_{M t}^{p}=\tau_{1}^{p} \dot{\theta}_{1}^{p}+\tau_{2}^{p} \dot{\theta}_{2}^{p}=\frac{\mathrm{d} E_{1}^{p}}{\mathrm{~d} t}+\frac{\mathrm{d} E_{p}^{p}}{\mathrm{~d} t}+\frac{\mathrm{d} E_{2}^{p}}{\mathrm{~d} t}+\frac{\mathrm{d} E_{3}^{p}}{\mathrm{~d} t},
$$

where $E_{1}^{p}, E_{p}^{p}, E_{2}^{p}$, and $E_{3}^{p}$ represent the mechanical energy of link $O O^{\prime}$, the moving platform, and the upper and the lower parts of the extendible link in the parallel solar tracker, respectively. Motor 1 is assumed to drive link $O O^{\prime}$. When motor 1 works as a generator and motor 2 works normally, the mechanical power can be expressed as

$$
P_{M t}^{p}=0+\tau_{2}^{p} \dot{\theta}_{2}^{p}=\frac{\mathrm{d} E_{1}^{p}}{\mathrm{~d} t}+\frac{\mathrm{d} E_{p}^{p}}{\mathrm{~d} t}+\frac{\mathrm{d} E_{2}^{p}}{\mathrm{~d} t}+\frac{\mathrm{d} E_{3}^{p}}{\mathrm{~d} t}-\tau_{1}^{p} \dot{\theta}_{1}^{p} .
$$


The mechanical power is equal to zero when the two motors of the parallel solar tracker work as generators. The serial solar tracker needs additional mechanical energy because of the large diameter of link $O O^{\prime}$. However, this requirement can be ignored due to the low speed of the solar tracker. Thus, the following can be considered:

$$
\frac{\mathrm{d} E_{1}^{p}}{\mathrm{~d} t}+\frac{\mathrm{d} E_{p}^{p}}{\mathrm{~d} t}=\frac{\mathrm{d} E_{1}^{s}}{\mathrm{~d} t}+\frac{\mathrm{d} E_{p}^{s}}{\mathrm{~d} t},
$$

where $E_{1}^{s}$ and $E_{p}^{s}$ represent the mechanical energy of link $O O^{\prime}$ and the moving platform in the serial solar tracker, respectively. Similarly, the mechanical power of serial solar tracker can be determined. Thus, the difference of mechanical power between the two solar trackers can be expressed as

$$
\Delta P_{M t}=\frac{\mathrm{d}}{\mathrm{d} t}\left(E_{2}^{p}+E_{3}^{p}\right)-k_{1}^{p} \tau_{1}^{p} \dot{\theta}_{1}^{p}-k_{2}^{p} \tau_{2}^{p} \dot{\theta}_{2}^{p}+k_{1}^{s} \tau_{1}^{s} \dot{\theta}_{1}^{s}+k_{2}^{s} \tau_{2}^{s} \dot{\theta}_{2}^{s}
$$

where $k_{i}$ is a parameter that depends on the working condition of motor $i$. When motor $i$ works as a generator, $k_{i}=1$. Otherwise, it equals 0 .

By integrating equation (44), the mechanical energy difference between the parallel solar tracker and its corresponding serial solar tracker can be written as

$$
\Delta W^{\{1\}}=\Delta E_{2,3}-\sum_{i=1,2} \min \left\{0, W_{i}^{p}\right\}+\sum_{i=1,2} \min \left\{0, W_{i}^{s}\right\},
$$

where $W_{1}^{p}, W_{2}^{p}, W_{1}^{s}$, and $W_{2}^{s}$ represent the mechanical energy output or absorbed by the motors of the parallel and serial solar trackers, respectively. $\Delta E_{2,3}$ represents the mechanical energy change of the extendible link.

The difference of the mechanical energy can also be calculated by the mechanical energy consumed by the motors, and it can be expressed as

$$
\Delta W^{\{2\}}=\sum_{i=1,2} \max \left\{0, W_{i}^{p}\right\}-\sum_{i=1,2} \max \left\{0, W_{i}^{s}\right\} .
$$

In the morning, when $E_{2}^{p}+E_{3}^{p}$ is reduced at a rate greater than the one at which one motor absorbs mechanical energy, that is, $(\mathrm{d} / \mathrm{d} t)\left(E_{2}^{p}+E_{3}^{p}\right)<k_{1}^{p} \tau_{1}^{p} \dot{\theta}_{1}^{p}+k_{2}^{p} \tau_{2}^{p} \dot{\theta}_{2}^{p}-k_{1}^{s} \tau_{1}^{s} \dot{\theta}_{1}^{s}-k_{2}^{s} \tau_{2}^{s}$ $\dot{\theta}_{2}^{s}<0$, the mechanical power input by the other motor to the solar tracker is smaller. In extreme cases, both motors are necessary to absorb the excessive mechanical energy released by the extendible link. When $k_{1}^{p} \tau_{1}^{p} \dot{\theta}_{1}^{p}+k_{2}^{p} \tau_{2}^{p} \dot{\theta}_{2}^{p}-k_{1}^{s} \tau_{1}^{s} \dot{\theta}_{1}^{s}-$ $k_{2}^{s} \tau_{2}^{s} \dot{\theta}_{2}^{s}<(\mathrm{d} / \mathrm{d} t)\left(E_{2}^{p}+E_{3}^{p}\right)<0$, one motor needs to increase the mechanical power to drive link $O O^{\prime}$ and the moving platform.

\section{Performance Comparison of Two Solar Trackers}

5.1. Geometrical and Inertial Parameters. In this paper, the energy consumption of the proposed solar tracker and its corresponding serial solar tracker with the extendible link removed are compared. It is assumed that the two solar trackers have the same static stiffness due to the low speed of the solar tracker for a fair comparison. Here, the diameter of link $O O^{\prime}$ in the serial solar tracker is modified to make the serial solar tracker have the same static stiffness as the proposed solar tracker. The deformation of the mechanism will prevent the parabolic dish concentrator from performing accurate tracking. Thus, the average value of the angular error between the parabolic axis and the sunlight is regarded as the stiffness evaluation index.

Based on the structural matrix method [14], the stiffness model of the solar tracker is derived and then the value of stiffness evaluation index can be obtained. Figure 4 shows the angular error $\Delta \theta$ in the parallel solar tracker, where $n$ is the direction of the parabolic axis. The serial solar tracker is similar to cantilever beam structure and thus has a poor rigidity, and the stiffness of the proposed solar tracker with parallel mechanism increases through the extendible link. Stiffness analysis shows that the diameter of link $O O^{\prime}$ of the serial solar tracker should be increased by $4 \%$ to make the proposed solar tracker and its corresponding serial solar tracker have the same static stiffness. The geometrical and inertial parameters of solar trackers are given in Table 2, where $r_{p}$ represents the radius of link $O O^{\prime}$ of the parallel solar tracker, and $r_{s}$ represents that of the serial solar tracker; and $r_{1}$ and $r_{2}$ are the radii of the upper and lower parts of the extendible link, respectively.

5.2. Workspace Analysis. Workspace is a very important index for a solar tracker. The singularity should be avoided in the workspace. For the serial solar tracker, the workspace is $-\pi<\alpha<\pi, 0 \leq \beta \leq \pi$ if the mechanism interference is not considered. However, the parallel solar tracker generally has many singular configurations. Thus, the workspace can be obtained based on the singularity analysis. The direct kinematic singularities and inverse kinematic singularities are generally identified when $|\mathbf{J}|=0$ and $|\mathbf{J}|=\infty$. The workspace without singularity can be determined upon identifying singularity, as shown in Figure 5.

Figure 5 shows that the parallel solar tracker has a relatively small workspace due to singular configuration. Daytime is the longest, and the elevation angle of the Sun is the largest during the summer solstice. The situation is completely opposite during the winter solstice. Thus, the area between the trajectory during the summer solstice and the trajectory during the winter solstice is the required workspace. The configuration outside the workspace cannot be reached, and the tracker can only be kept in a relatively close configuration in the workspace, reducing the absorption of solar energy to some extent. On the one hand, this condition is acceptable because the invalid trajectory space corresponds to early morning and evening. Due to the thickness, refraction, and scattering of the atmosphere, the radiant energy from the Sun to the Earth's surface is much less during these two periods. On the other hand, the geometrical parameters of the parallel solar tracker can be optimized to increase the workspace. 


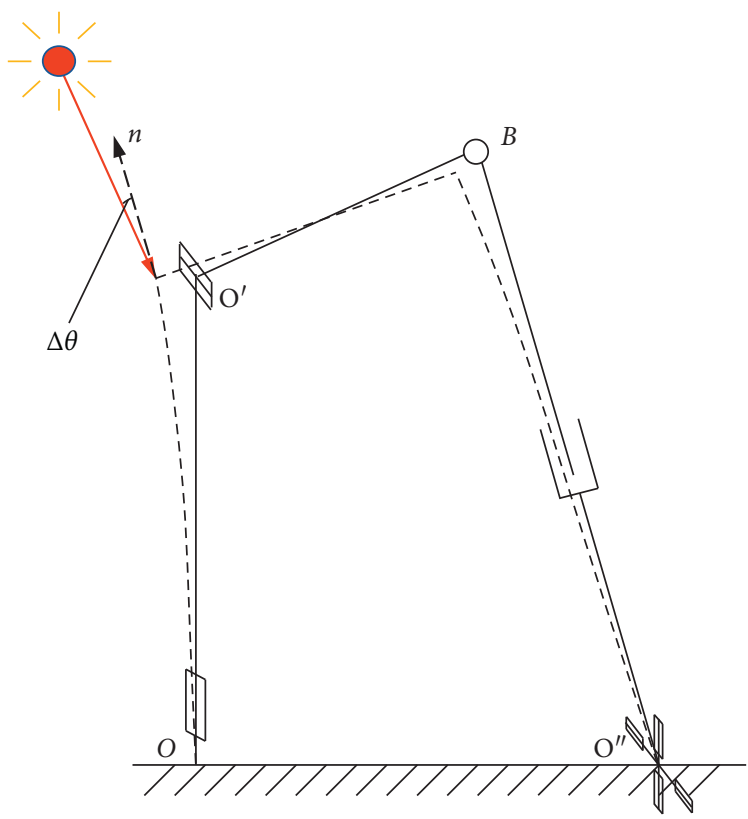

FIgURE 4: Angle error caused by the elastic deformation.

TABLe 2: Geometrical and inertial parameters.

\begin{tabular}{|c|c|}
\hline Parameters & Values \\
\hline $\begin{array}{llll}\mathbf{h} & \mathbf{a} & \mathbf{b}^{\prime} & \mathbf{r}_{p}^{\prime}(\mathrm{m})\end{array}$ & {$\left[\begin{array}{lll}0 & 0 & 1\end{array}\right]^{T} \quad\left[\begin{array}{lll}0 & 0.5 & 0\end{array}\right]^{T} \quad\left[\begin{array}{lll}0 & 0.5 & 0.05\end{array}\right]^{T} \quad\left[\begin{array}{lll}0 & 0 & 0.1\end{array}\right]^{T}$} \\
\hline$l_{2} l_{3} r_{p} r_{s} r_{1} r_{2}(\mathrm{~m})$ & $\begin{array}{lllllll}10.8 & 0.8 & 0.5 & 0.52 & 0.06 & 0.08\end{array}$ \\
\hline$m_{1}^{p} m_{1}^{s} m_{2} m_{3} m_{p}(\mathrm{~kg})$ & $\begin{array}{lllll}62.0465 & 67.1094 & 71.4775 & 55.5936 & 100\end{array}$ \\
\hline $\mathbf{I}_{1}^{P^{1}}\left(\mathrm{~kg} \cdot \mathrm{m}^{2}\right)$ & $\operatorname{diag}\left(\begin{array}{lll}5.1705 & 5.1705 & 0.0776\end{array}\right)$ \\
\hline $\mathbf{I}_{1}^{s}\left(\mathrm{~kg} \cdot \mathrm{m}^{2}\right)$ & $\operatorname{diag}\left(\begin{array}{lll}5.5925 & 5.5925 & 0.0907\end{array}\right)$ \\
\hline $\mathbf{I}_{2}\left(\mathrm{~kg} \cdot \mathrm{m}^{2}\right)$ & $\operatorname{diag}\left(\begin{array}{lll}3.8121 & 3.8121 & 0.1287\end{array}\right)$ \\
\hline $\mathbf{I}_{3}\left(\mathrm{~kg} \cdot \mathrm{m}^{2}\right)$ & $\operatorname{diag}\left(\begin{array}{lll}2.9650 & 2.9650 & 0.2780\end{array}\right)$ \\
\hline $\mathbf{I}_{p}\left(\mathrm{~kg} \cdot \mathrm{m}^{2}\right)$ & $\operatorname{diag}\left(\begin{array}{lll}200 & 200 & 200\end{array}\right)$ \\
\hline
\end{tabular}

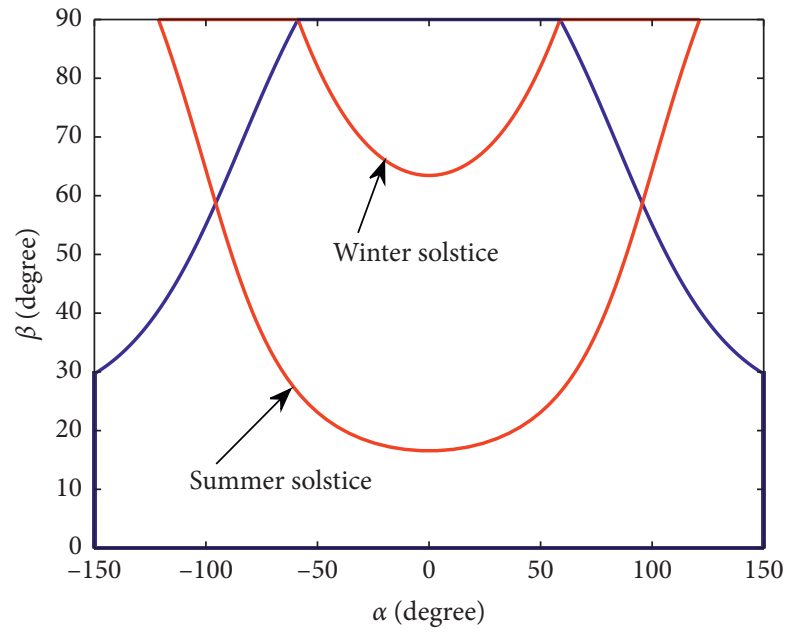

- Workspace of UPS-RR

Trajectory envelope of the sun

Figure 5: Workspace of the mechanism and trajectory envelope of the Sun. 
5.3. Torque Analysis. The motion trajectory of the solar tracker is obtained by equation (7); the torques of the parallel solar tracker and its corresponding serial solar tracker can be computed, as shown in Figure 6. From Figures 6(a) and 6(c), one may see that the driving torque of link $O O^{\prime}$ in the parallel solar tracker is much larger than that in the serial solar tracker, and sometimes it is a resistance torque. The extendible link provides a large torque to drive the moving platform. Thus, a resistance torque is required to limit the angular acceleration.

The load of motor 1 in the serial solar tracker only includes the inertia moment of link $O O^{\prime}$ and the moving platform during the rotation process. The solar tracker moves slowly, and the angular acceleration of the joint is very small. Thus, the driving torque that drives link $O O^{\prime}$ of the serial solar tracker is very small.

Figure 6(b) shows that the torque driving the extendible link is very small. Figure $6(\mathrm{~d})$ shows that the torque driving the moving platform in the serial solar tracker is larger and more stable in January and December than in other months because the elevation angle of the Sun is small in winter. The mass center of the mirror does not pass through the axis of link $O O^{\prime}$. Thus, the driving torque is always opposite to the gravity moment. At noon in summer, the driving torques provided by motor 2 of the proposed and the corresponding serial solar trackers are the largest because the elevation angle of the Sun is maximum, and the gravity torque is minimum at this time. In contrast to the serial solar tracker, the torque driving the extendible link is not always in the same direction. Thus, the force of the extendible link against the moving platform is not always used to overcome the mirror's gravity. At noon in summer, the motor needs to overcome the difference between the gravity moment of the moving platform and the extendible link to drive the moving platform.

5.4. Electrical Energy Analysis. Electrical energy greatly contributes to the total energy consumption of the lowspeed solar tracker. Thus, the electrical energy is also analyzed. Delta ECM-A3LC10807 servo motor is used in the solar tracker, and the motor parameters are given in Table 3. The power loss can be reduced only by increasing the reduction ratio of active joints. However, the reduction ratio cannot be too big. Otherwise, the mechanical efficiency is debased. The reduction ratio of each drive joint needs to ensure that the maximum driving torque does not exceed the maximum torque of the motor.

The daily energy consumption caused by resistance and inductance is shown in Figure 7. The electrical energy consumption of the parallel solar tracker is significantly lower than that of the serial solar tracker every day. The annual electrical energy consumptions of the proposed solar tracker and its corresponding serial solar tracker are 19707 and $109390 \mathrm{~kJ}$, respectively. Thus, the total energy consumption is reduced by $81.98 \%$.
5.5. Mechanical Energy Analysis. Based on equation (40), the consumed mechanical energy of the parallel solar tracker and its corresponding serial solar tracker can be computed, as shown in Figure 8 . Figures 7 and 8 show that the consumed mechanical energy is significantly smaller than the consumed electrical energy. The proposed solar tracker consumes lower mechanical energy in spring and autumn than the serial solar tracker. For the full year, the mechanical energy consumption of the parallel solar tracker is $51.142 \mathrm{~kJ}$, which is $7.55 \%$ less than that of the serial solar tracker. Thus, the total mechanical energy consumption is reduced though the extendible link $O O^{\prime}$ is introduced.

Figure 9 shows the mechanical energy of moving parts in the parallel solar tracker. The mechanical energy of the extendible link reduces in the morning and increases in the afternoon, whereas that of link $O O^{\prime}$ and moving platform changes in the opposite tendency. The kinetic energy of each component is almost equal to 0 due to the low speed, so the change of mechanical energy is equivalent to the change of gravitational potential energy. The results in Figure 9 show that there is energy transfer between the components of the mechanism at any time. In spring and autumn, the energy transfer efficiency between different components is high, and the gravity is used to drive the mechanism more effectively instead of being the load on the motor. Therefore, the parallel solar tracker consumes less energy than its corresponding serial solar tracker in summer and autumn.

Figures 10 and 11 show the mechanical power difference of two solar trackers and the mechanical power of each motor at any time on any day in a year, respectively. Figure 10 shows that the mechanical power of the parallel solar tracker is smaller than its corresponding serial solar tracker in most time of the morning. In this case, at least one motor in the parallel solar tracker works as a generator, which can be found in Figures 11(a) and 11(b), and the mechanical energy released by the extendible link is more than that absorbed by one or two motors, which corresponds to the case of $(\mathrm{d} / \mathrm{d} t)\left(E_{2}^{p}+E_{3}^{p}\right)<k_{1}^{p} \tau_{1}^{p} \dot{\theta}_{1}^{p}+k_{2}^{p} \tau_{2}^{p} \dot{\theta}_{2}^{p}-k_{1}^{s} \tau_{1}^{s} \dot{\theta}_{1}^{s}-k_{2}^{s} \tau_{2}^{s}$ $\dot{\theta}_{2}^{s}<0$ in Section 4. At other times of the morning, the other motor needs to increase the mechanical power to make link $O O^{\prime}$ and the moving platform get enough mechanical energy, which corresponds to the case of $k_{1}^{p} \tau_{1}^{p} \dot{\theta}_{1}^{p}+k_{2}^{p} \tau_{2}^{p} \dot{\theta}_{2}^{p}-$ $k_{1}^{s} \tau_{1}^{s} \dot{\theta}_{1}^{s}-k_{2}^{s} \tau_{2}^{s} \dot{\theta}_{2}^{s}<(\mathrm{d} / \mathrm{d} t)\left(E_{2}^{p}+E_{3}^{p}\right)<0$ in Section 4. In the afternoon, the serial solar tracker does not consume mechanical energy. Moreover, the mechanism is driven by gravity, and the output mechanical energy is absorbed by the motor. The mechanical energy released by link $O O^{\prime}$ and the moving platform is not enough to drive the parallel solar tracker. Thus, the parallel solar tracker requires additional mechanical energy in the afternoon.

Therefore, in comparison with the serial solar tracker, the proposed solar tracker does not necessarily consume additional mechanical energy even if the extendible link is added. Two main factors affect mechanical energy. One is the "counterweight effect." From the energy point of view, the mutual 


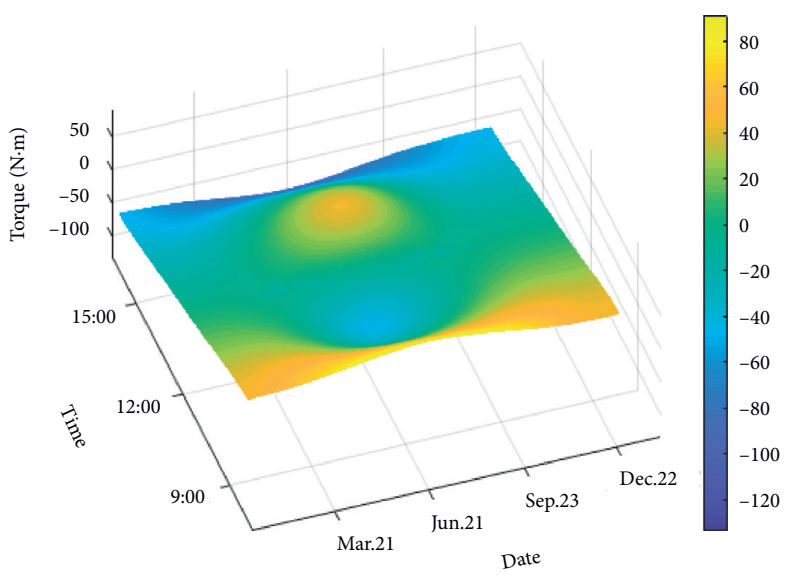

(a)

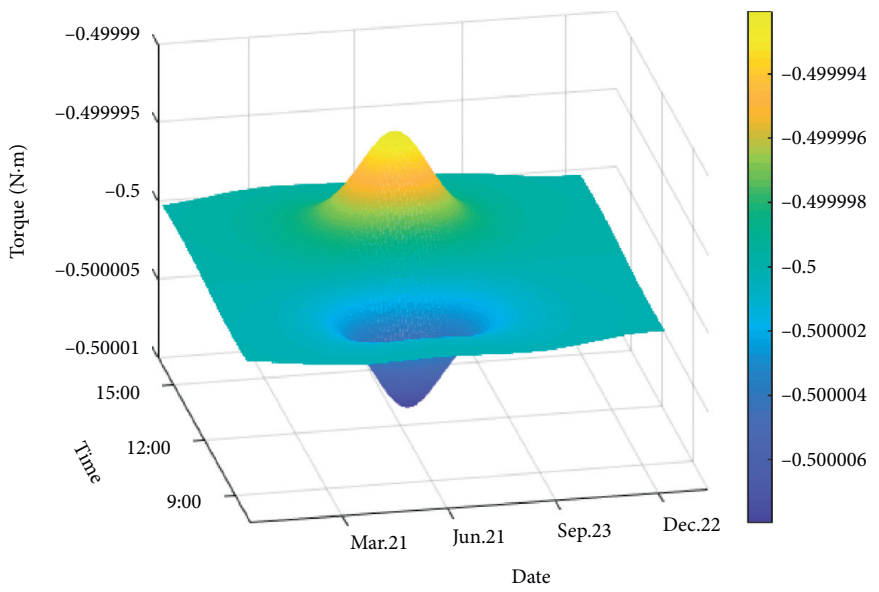

(c)

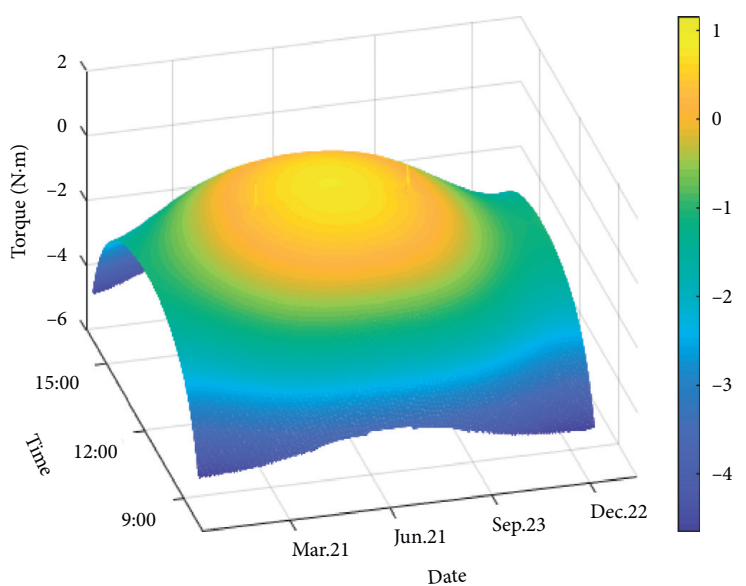

(b)

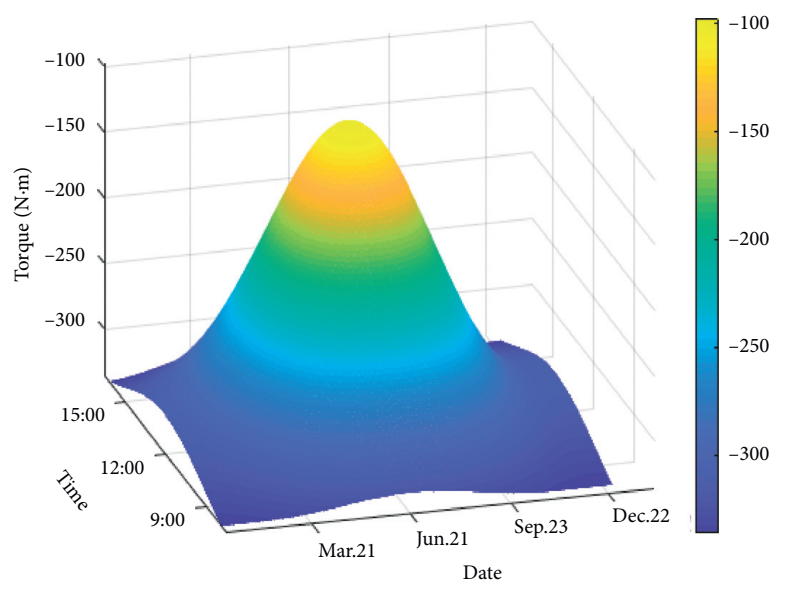

(d)

FiguRe 6: Driving torque. (a) Driving torque of motor 1 in parallel tracker. (b) Driving torque of motor 2 in parallel tracker. (c) Driving torque of motor 1 in serial tracker. (d) Driving torque of motor 2 in serial tracker.

TAble 3: Parameters of Delta ECM-A3LC10807 servo motor.

\begin{tabular}{lc}
\hline Parameters & Values \\
\hline$R(\Omega)$ & 0.6 \\
$L(\mathrm{mH})$ & 4.6 \\
$K_{T}(\mathrm{~N} \cdot \mathrm{m} / \mathrm{A})$ & 0.469 \\
$K_{E}(\mathrm{mV} \cdot \mathrm{min} / \mathrm{r})$ & 17 \\
\hline
\end{tabular}

conversion of energy between components reduces the input of mechanical energy. The mechanical energy required for the motion of extendible link can be partially provided by link $O O^{\prime}$ and the moving platform. The extendible link can store the energy released by link $O O^{\prime}$ and moving platform. When link $O O^{\prime}$ and the moving platform need energy, the extendible link can release the energy again. The other factor is that the motor sometimes works as a generator, and certain mechanical energy is input into the motor and converted to thermal energy. Therefore, the proposed solar tracker consumes less mechanical energy than the corresponding serial solar tracker though an additional extendible link is introduced.

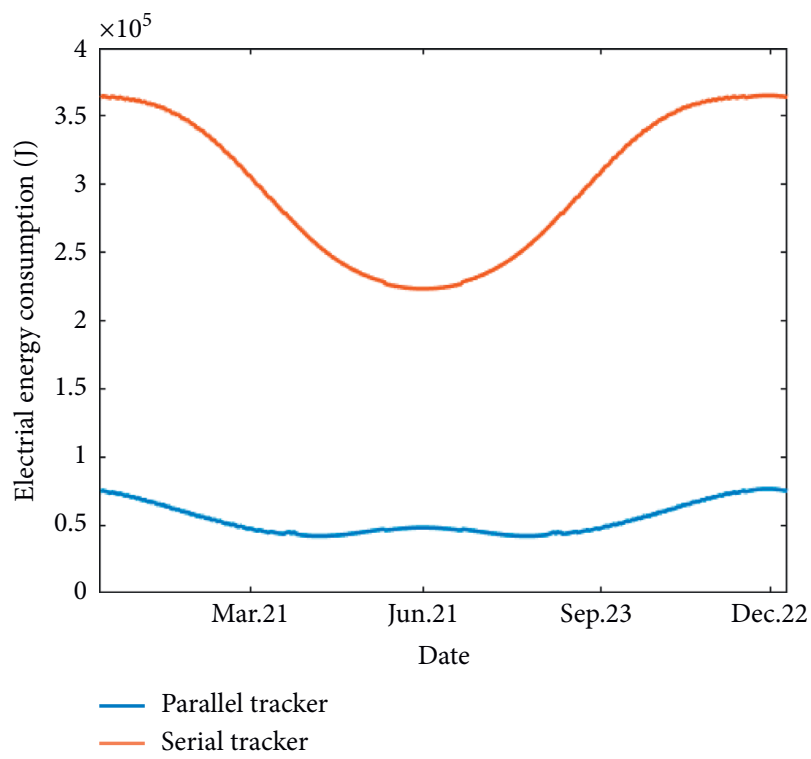

Figure 7: Daily energy consumption. 


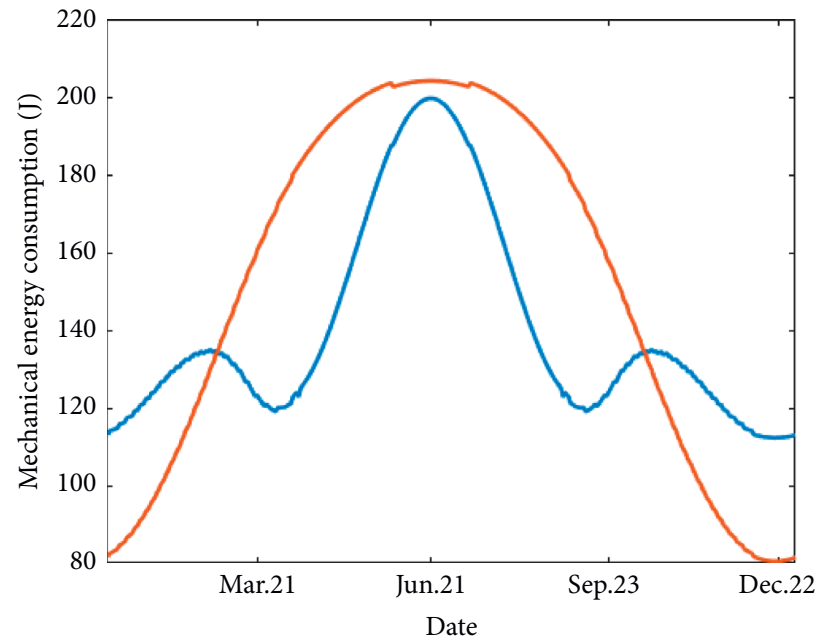

— Parallel tracker

— Serial tracker

FIgURE 8: Mechanical energy consumption.

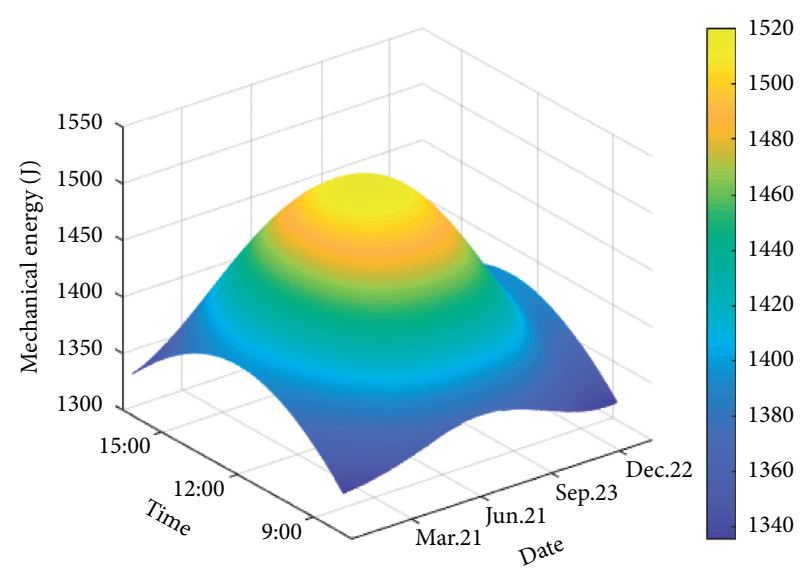

(a)

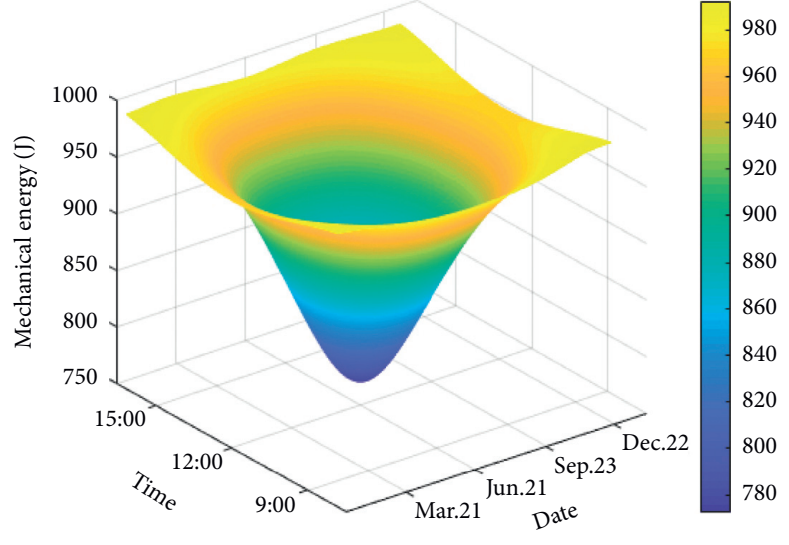

(b)

FIGURE 9: Mechanical energy of different part of parallel solar tracker. (a) Link $O O^{\prime}$ and moving platform. (b) Extendible link.

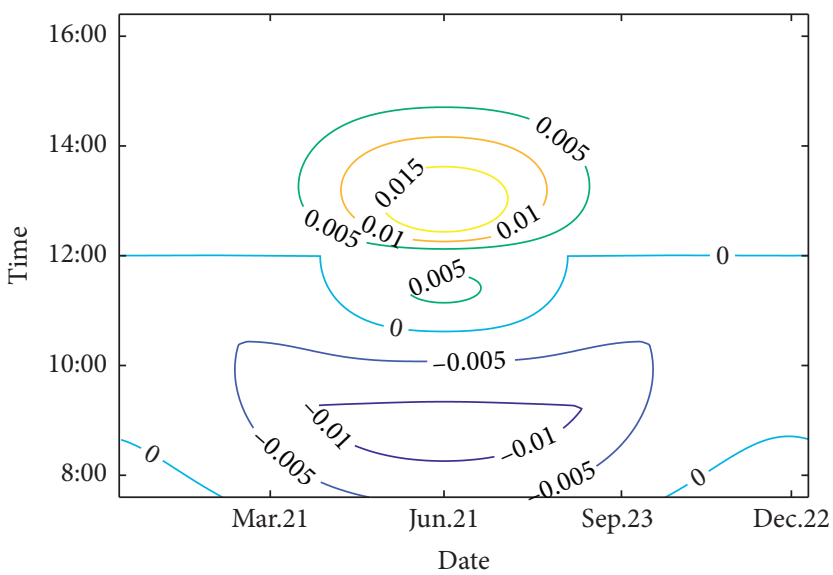

Figure 10: Contour of the mechanical power difference. 


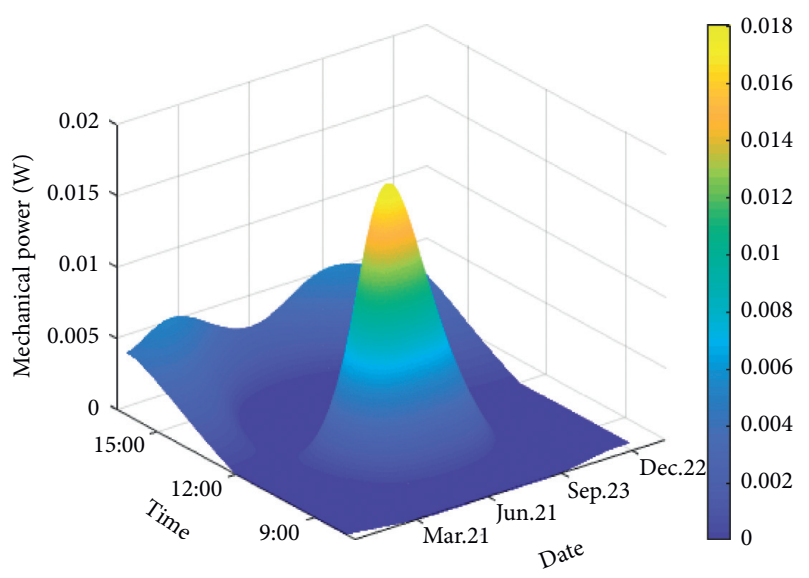

(a)

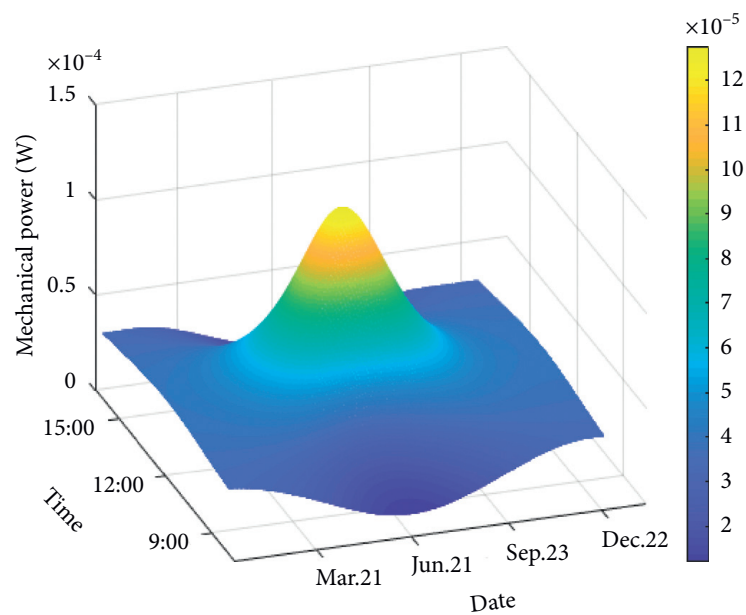

(c)

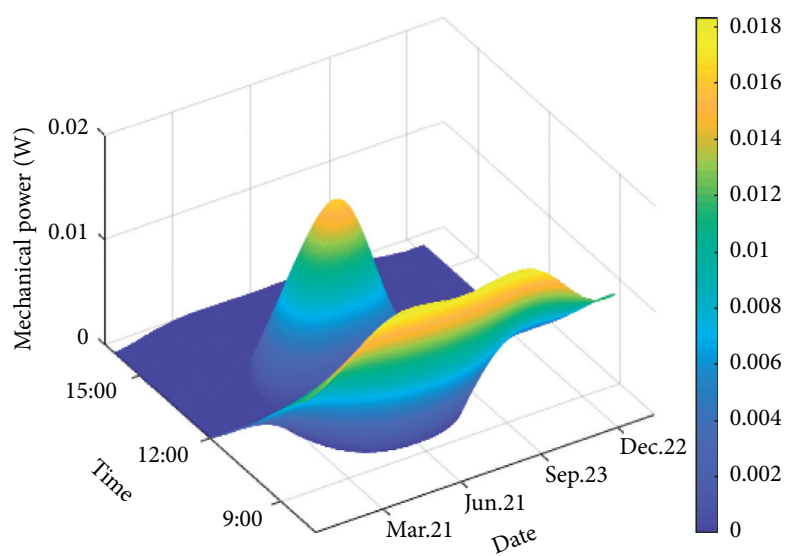

(b)

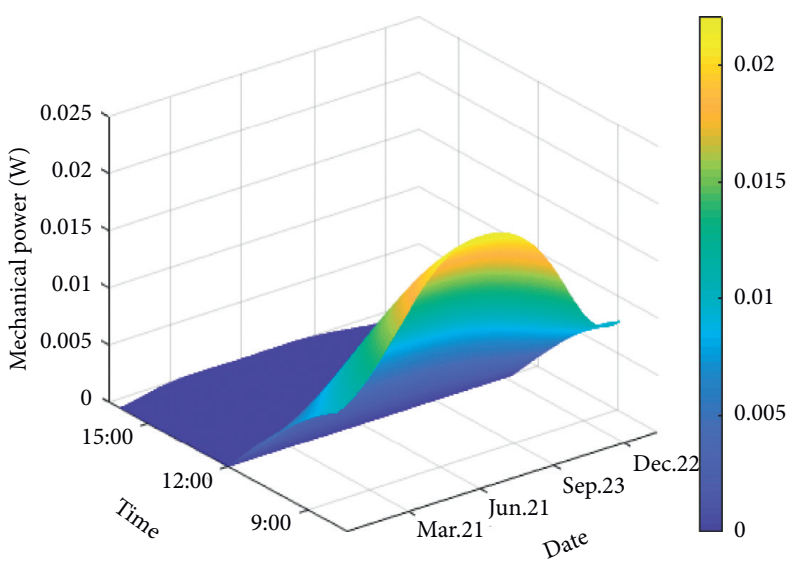

(d)

FiguRE 11: Mechanical power of each motor. (a) Motor 1 in parallel solar tracker. (b) Motor 2 in parallel solar tracker. (c) Motor 1 in serial solar tracker. (d) Motor 2 in serial solar tracker.

\section{Conclusions}

This paper proposes a new parallel solar tracker, and its energy consumption is investigated. The energy consumptions of the proposed solar tracker and its corresponding serial solar tracker with the extendible link removed are compared. With the same motion, the mechanical energy consumption of the proposed solar tracker is reduced by $7.55 \%$. The mechanical energy between different components can be converted during the movement. The motor of the proposed solar tracker has a long stroke and small driving force. Thus, the current is small at the same reduction ratio, and the total energy consumption, including mechanical and electrical energy, caused by the resistance and inductance is reduced by $81.95 \%$. The solar tracker shall be considered acceptable in practice.

In this study, some iron loss in the calculation of instantaneous power and the influence of weather changes on the trajectory are not considered. In the further work, these factors will be considered.

\section{Data Availability}

The data used to support the findings of this study are included within the article.

\section{Conflicts of Interest}

The authors declare that they have no conflicts of interest.

\section{Acknowledgments}

This work was supported by the National Key Research and Development Program of China (no. 2017YFE0111300) and EU H2020-MSCA-RISE-ECSASDPE (no. 734272).

\section{References}

[1] C.-Y. Lee, P.-C. Chou, C.-M. Chiang, and C.-F. Lin, "Sun tracking systems: a review," Sensors, vol. 9, no. 5, pp. 3875-3890, 2009. 
[2] K. Lovegrove, G. Burgess, and J. Pye, "A new $500 \mathrm{~m}^{2}$ paraboloidal dish solar concentrator," Solar Energy, vol. 85, no. 4, pp. 620-626, 2011.

[3] K. Azizi and A. Ghaffari, "Design and manufacturing of a high-precision sun tracking system based on image processing," International Journal of Photoenergy, vol. 2013, Article ID 754549, 7 pages, 2013.

[4] O. Altuzarra, I. Seras, E. Macho, and J. Aginaga, A Low Energy Consumption Solar Tracker Based in Parallel Kinematics, in Romansy 19-Robot Design, Dynamics and Control, Springer, Vienna, Austria, 2013.

[5] A. K. Saymbetov, M. K. Nurgaliyev, Y. Tulkibaiuly et al., "Method for increasing the efficiency of a biaxial solar tracker with exact solar orientation," Applied Solar Energy, vol. 54, no. 2, pp. 126-130, 2018.

[6] E. Lorenzo, M. Pérez, A. Ezpeleta, and J. Acedo, "Design of tracking photovoltaic systems with a single vertical axis," Progress in Photovoltaics: Research and Applications, vol. 10, no. 8, pp. 533-543, 2002.

[7] C. S. Chin, A. Babu, and W. McBride, "Design, modeling and testing of a standalone single axis active solar tracker using MATLAB/simulink," Renewable Energy, vol. 36, no. 11, pp. 3075-3090, 2011.

[8] C. Alexandru and C. Pozna, "Different tracking strategies for optimizing the energetic efficiency of a photovoltaic system," in Proceedings of the 2008 IEEE International Conference on Automation, Quality and Testing, Robotics, vol. 3, pp. 434439, IEEE, Cluj-Napoca, Romania, May 2008.

[9] F. G. Gil, M. D. S. Martin, J. P. Vara, and J. R. Calvo, “A reviewof solar tracker patents in Spain," Energy Problems and Environmental Engineering, L. Perlovsky, D. D. Dionysiou, L. A. Zadeh et al., Eds., pp. 292-29, World Scientific and Engineering Academy and Society, Athens, Greece, 2009.

[10] L. Barker, M. Neber, and H. Lee, "Design of a low-profile twoaxis solar tracker,” Solar Energy, vol. 97, pp. 569-576, 2013.

[11] H. Mousazadeh, A. Keyhani, A. Javadi, H. Mobli, K. Abrinia, and A. Sharifi, "A review of principle and sun-tracking methods for maximizing solar systems output," Renewable and Sustainable Energy Reviews, vol. 13, no. 8, pp. 1800-1818, 2009.

[12] T. Tomson, "Discrete two-positional tracking of solar collectors," Renewable Energy, vol. 33, no. 3, pp. 400-405, 2008.

[13] R. Eke and A. Senturk, "Performance comparison of a doubleaxis sun tracking versus fixed PV system," Solar Energy, vol. 86, no. 9, pp. 2665-2672, 2012.

[14] P. Roth, A. Georgiev, and H. Boudinov, "Design and construction of a system for sun-tracking," Renewable Energy, vol. 29, no. 3, pp. 393-402, 2004.

[15] A. K. Saymbetov, M. K. Nurgaliyev, Y. D. Nalibayev et al., "Intelligent energy efficient wireless communacation system for street lighting," in Proceedings of the 2018 International Conference on Computing and Network Communications (CoCoNet), pp. 18-22, Astana, Kazakhstan, August 2018.

[16] J. Wu, G. Yu, Y. Gao, and L. Wang, "Mechatronics modeling and vibration analysis of a 2-DOF parallel manipulator in a 5DOF hybrid machine tool," Mechanism and Machine Theory, vol. 121, pp. 430-445, 2018.

[17] J. Wu, J. Wang, L. Wang, and T. Li, "Dynamics and control of a planar 3-DOF parallel manipulator with actuation redundancy," Mechanism and Machine Theory, vol. 44, no. 4, pp. 835-849, 2009.

[18] A. Battezzato, S. Mauro, and C. Scarzella, "Developing a parallel kinematic solar tracker for HCPV," in Proceedings of the ASME 2012 11th Biennial Conference on Engineering
Systems Design and Analysis, pp. 459-464, American Society of Mechanical Engineers (ASME), New York, NY, USA, July 2012.

[19] O. Altuzarra, I. Seras, E. Macho, and J. Aginaga, "A low energy consumption solar tracker based in parallel kinematics," in Proceedings of the Romansy 19-Robot Design, Dynamics and Control, pp. 69-76, Springer, Vienna, Austria, January 2013.

[20] O. Altuzarra, E. Macho, J. Aginaga, and V. Petuya, "Design of a solar tracking parallel mechanism with low energy consumption," Proceedings of the Institution of Mechanical Engineers, Part C: Journal of Mechanical Engineering Science, vol. 229, no. 3, pp. 566-579, 2015.

[21] J. Wu, X. Chen, and L. Wang, "Design and dynamics of a novel solar tracker with parallel mechanism," IEEE/ASME Transactions on Mechatronics, vol. 21, no. 1, pp. 88-97, 2015.

[22] D. Zhang and B. Wei, "Dynamic balancing of robotic mechanisms via reconfiguration and integration design," International Journal of Robotics \& Automation, vol. 32, no. 6, pp. 551-559, 2017.

[23] H. Tang, D. Zhang, S. Guo, H. Qu, and G. Huang, "Kinematics analysis of a novel 2R1T parallel mechanism," International Journal of Robotics \& Automation, vol. 33, no. 2, pp. 127-140, 2018.

[24] Z. Yang and D. Zhang, "Energy optimal adaption and motion planning of a 3-RRS banlanced manipulator," International Journal of Robotics \& Automation, vol. 34, no. 5, pp. 518-525, 2019.

[25] A. Cammarata, "Optimized design of a large-workspace 2DOF parallel robot for solar tracking systems," Mechanism and Machine Theory, vol. 83, pp. 175-186, 2015.

[26] J. Wu, J. Wang, and Z. You, "An overview of dynamic parameter identification of robots," Robotics and ComputerIntegrated Manufacturing, vol. 26, no. 5, pp. 414-419, 2010.

[27] R. Ramkumar, C. Karthikeyan, and A. K. Dash, "A new workspace analysis method for 6-DOF 3-RRRS parallel manipulators," International Journal of Robotics \& Automation, vol. 34, no. 2, pp. 135-145, 2019.

[28] X. Du, Y. Li, P. Wang, Z. Ma, D. Li, and C. Wu, "Design and optimization of solar tracker with u-pru-pus parallel mechanism," Mechanism and Machine Theory, vol. 155, Article ID 104107, 2021.

[29] J. Wu, B. Zhang, and L. Wang, "Optimum design and performance comparison of a redundantly actuated solar tracker and its nonredundant counterpart," Solar Energy, vol. 127, pp. 36-47, 2016.

[30] A. Saymbetov, "Optimized single-axis schedule solar tracker in different weather conditions," Energies, vol. 13, no. 19, p. 5226, 2020.

[31] N. Kuttybay, S. Mekhilef, A. Saymbetov, M. Nurgaliyev, and Z. Kopzhan, "An automated intelligent solar tracking control system with adaptive algorithm for different weather conditions," in Proceedings of the 2019 IEEE International Conference on Automatic Control and Intelligent Systems (I2CACIS), IEEE, Shah Alam, Malaysia, June 2019.

[32] R. Ur-Rehman, S. Caro, D. Chablat, and P. Wenger, "Multiobjective path placement optimization of parallel kinematics machines based on energy consumption, shaking forces and maximum actuator torques: application to the orthoglide," Mechanism and Machine Theory, vol. 45, no. 8, pp.1125-1141, 2010. 